\title{
Aesthetic and functional outcome after breast conserving surgery - Comparison between conventional and oncoplastic resection
}

\author{
Ojala, K.
}

2017-04

Ojala , K , Meretoja , T J \& Leidenius , M H K 2017 , ' Aesthetic and functional outcome after breast conserving surgery - Comparison between conventional and oncoplastic resection ', European Journal of Surgical Oncology , vol. 43 , no. 4 , pp. 658-664 . https://doi.org/10.1016/j.ejso.2016.11.019

http://hdl.handle.net/10138/236951

https://doi.org/10.1016/j.ejso.2016.11.019

publishedVersion

Downloaded from Helda, University of Helsinki institutional repository.

This is an electronic reprint of the original article.

This reprint may differ from the original in pagination and typographic detail.

Please cite the original version. 


\title{
Aesthetic and functional outcome after breast conserving surgery - Comparison between conventional and oncoplastic resection
}

\author{
K. Ojala*, T.J. Meretoja, M.H.K. Leidenius \\ Breast Surgery Unit, Comprehensive Cancer Center, Helsinki University Hospital and Helsinki University, \\ Finland
}

Accepted 21 November 2016

Available online 18 December 2016

\begin{abstract}
Background: Recent studies implicate that oncoplastic breast cancer surgery provides better aesthetic outcome than conventional resection. Several factors have been associated with poor aesthetic outcome. This study aims to compare patient-reported aesthetic and functional outcome after conventional and oncoplastic resection and to evaluate prognostic factors for poor aesthetic outcome in a populationbased setting.

Methods: 637 patients having breast conserving treatment (BCT) due to unilateral primary breast cancer at a single hospital district during 2010 were included. Aesthetic and functional outcome were evaluated using two questionnaires three years after surgery.

Results: Questionnaires were returned by 379 (59\%) patients; 293 (77\%) of these had conventional and 86 (23\%) oncoplastic resection. Patients in oncoplastic resection group had larger tumour diameter $(\mathrm{p}<0.001)$, larger resection specimens $(\mathrm{p}<0.001)$, and more often multifocal tumours $(\mathrm{p}=0.032)$, node positive cancer $(\mathrm{p}=0.029)$ and lower quadrant tumour localization $(\mathrm{p}=0.007)$. Aesthetic outcome according to BCTOS questionnaire was good in $284(75 \%)$ patients; $52(61 \%)$ patients in the oncoplastic group and 230 patients $(81 \%)$ in the conventional resection group, $\mathrm{p}<0.001$. Larger tumour diameter $(\mathrm{p}=0.033)$, multifocality $(\mathrm{p}=0.022)$, weight of resection specimen $(<0.001)$ and oncoplastic surgery $(\mathrm{p}<0.001)$ were predicting poor aesthetic outcome, when all patients were included. Tumour multifocality $(\mathrm{p}=0.013)$ remained predictor of poor aesthetic outcome in conventional resection group but not in oncoplastic resection group. Conclusions: Patient satisfaction to aesthetic outcome after BCT is high. Conventional resection provides good aesthetic outcome in appropriately selected patients. Oncoplastic resection enables BCT in patients with larger and multifocal tumours with favourable aesthetic outcome.
\end{abstract}

(C) 2016 Elsevier Ltd, BASO The Association for Cancer Surgery, and the European Society of Surgical Oncology. All rights reserved.

\section{Introduction}

A good aesthetic outcome after breast conserving therapy (BCT) in breast cancer is important. Previous studies have demonstrated correlations between aesthetic outcome and quality of life (QoL). ${ }^{1-3}$ Several factors associated with poor aesthetic outcome have been identified: central, medial or inferior tumour locations, ${ }^{4,5}$ larger tumour size, ${ }^{4}$ larger specimen volume, ${ }^{5}$ re-operation due to positive margins, ${ }^{6}$ radiation therapy and radiation boost. ${ }^{7,8}$

\footnotetext{
* Corresponding author. Töölö Hospital, P.O. Box 266, 00029 HUS, Finland. Fax: +3589 47176301 .

E-mail address: kaisu.ojala@helsinki.fi (K. Ojala).
}

Various oncoplastic techniques have been developed to improve aesthetic outcome after BCT. Increasing number of studies confirm oncologic safety of oncoplastic breast conserving surgery. ${ }^{9-12}$ With oncoplastic techniques, patients with larger or multifocal tumours may become eligible for BCT rather than mastectomy, without compromising aesthetic outcome.

A recent meta-analysis ${ }^{10,13}$ implicates that oncoplastic breast surgery provides a better aesthetic outcome. However, also contradictory results have been reported. ${ }^{14}$ Moreover, evaluation of patient-reported aesthetic outcome is lacking a gold standard and current methods vary widely. ${ }^{13,15}$ Study instruments to evaluate aesthetic and 
functional outcome vary from a single question assessing to validated multi-item questionnaires. Previous studies also show that patient-reported aesthetic outcome questionnaires provide more favourable aesthetic outcomes than objective aesthetic outcome measurements. ${ }^{1,3}$

For these reasons, this study aims to compare patientreported aesthetic and functional outcome after conventional resection and oncoplastic resection and to evaluate possible prognostic factors for poor aesthetic outcome in a population-based setting.

\section{Patients and methods}

All patients having breast conserving surgery (BCS) due to primary breast cancer at the Helsinki and Uusimaa Hospital District during 2010 were included in this study; 664 patients were retrospectively identified from a database. 27 patients were excluded due to bi-lateral disease or earlier breast cancer. Remaining 637 patients constitute the study population. The data was completed with information from electronic patient records on tumour characteristics, complications and oncoplastic operation techniques, as well as information of radiation therapy. We explored the following characteristics:

- patient age

- tumour characteristics (histological type, tumour size, tumour multifocality, axillary nodal status, ER, PR, HER2 and Ki-67, tumour localization)

- surgical treatment characteristics (operation type, reexcision rate, resection volume, contralateral surgery, axillary procedures)

- complications requiring surgical treatment

- information of radiation therapy including tumour bed booster

Questionnaire forms and informed consent form were sent three years after surgery by mail, with prepaid return envelope included. In three years, sufficient time after radiation therapy has passed to visualize effects of irradiation and booster. $379(59 \%)$ patients agreed to take part in the study by returning questionnaires and signed informed consent form. The study plan was approved by the Ethics Committee of Helsinki University Central Hospital.

\section{Aesthetic and functional outcome questionnaires}

Aesthetic and functional outcomes were evaluated with the use of two separate questionnaires. Breast Cancer Treatment Outcome Scale (BCTOS) was designed to assess woman's subjective evaluation of the aesthetic and functional outcome after breast cancer treatment. ${ }^{16}$ It addresses many important dimensions of after-treatment morbidity with respect to aesthetic and functional outcome and specifically targets the surgical aspects of BCT. We translated BCTOS questionnaire into Finnish and Swedish, since - to our knowledge - it has not been used in Finland before. Patients rate different items according to symmetry between the treated and the untreated breast on a four-point scale (from $1=$ no difference between treated and untreated breast to $4=$ large difference between treated and untreated breast). BCTOS consists of three internally consistent scales, which are defined as Aesthetic, Functional and Sensitivity status. The score value of each subscale is the mean of items belonging to this scale. We defined BCTOS aesthetic status cut off value $\geq 3$ to be a poor aesthetic outcome. BCTOS aesthetic score from 2.0 to 2.99 was considered intermediate and score $<2$ as good aesthetic outcome. For comparing individual items between conventional and aesthetic groups, four-point scale was categorized into two groups for statistical analyses, so that alternatives 1 and 2 (good or excellent outcome) were combined as well as alternatives 3 and 4 (intermediate or poor outcome).

Another, author-created questionnaire was focussing on aesthetic and functional outcome of the operated breast. In this five-point questionnaire patients were asked to grade aesthetic outcome of operated breast in general and in some specific areas such as breast size, nipple appearance and position, breast shape and position, scar tissue, fitting of bra and symmetry to contralateral breast. Questionnaire also asked if patients had any problems in everyday life due to the aesthetic outcome.

\section{Surgical techniques}

In this study, oncoplastic resection refers to levels 1 and 2 oncoplastic procedures. ${ }^{17}$ The phrase "conventional breast resection" is used when resection of breast tissue is performed with adequate mobilization and closure of tissue to reach best possible aesthetic outcome with incision over tumour and excision of skin. In fact, our conventional breast resection technique can be considered as level 1 oncoplastic procedure. One of the reported oncoplastic techniques, that is extensive dual plane undermining, in turn, includes much more extensive undermining both in the fascia and subcutaneous level.

\section{Statistical methods}

Statistical analyses were performed with SPSS Statistics software version 22.0 (IBM corporation, Somers, NY). Baseline comparisons between oncoplastic and conventional resection groups were performed using chi-squared test and medians of continuous variables were compared using the Mann-Whitney $U$ and Kruskal-Wallis tests.

\section{Results}

\section{Patient and tumour characteristics}

Questionnaire was returned by 379 (59\%) patients; 293 (77\%) of these patients had conventional and $86(23 \%)$ 
oncoplastic resection. The proportion of conventional and oncoplastic procedures in the whole study population was similar, $79 \%$ and $21 \%$, respectively. Patient and tumour characteristics are displayed in Table 1. Patients in the oncoplastic resection group had significantly larger tumour diameter $(\mathrm{p}<0.001)$, larger resection specimens $(\mathrm{p}<0.001)$, and more often multifocal tumours $(\mathrm{p}=0.032)$, node positive cancer $(\mathrm{p}=0.029)$ as well as lower quadrant tumour localization $(\mathrm{p}=0.007)$. Axillary lymph node dissection was performed significantly more often in the oncoplastic resection group $(\mathrm{p}=0.007)$ reflecting difference in nodal status between groups. There was no statistical difference between groups in receiving radiation therapy and tumour bed booster (Table 1).

\section{Surgery}

Oncoplastic resection techniques were used as follows: racket mammoplasty $19(22 \%)$, reduction mammoplasty techniques $19(22 \%)$, round block $16(19 \%)$, rotationplasty techniques 16 (19\%), extensive dual plane undermining 12 (14\%) and other oncoplastic techniques 4 (5\%). Contralateral breast symmetry procedure was performed in $20(23 \%)$ patients in the oncoplastic group compared to $3(1 \%)$ in the conventional resection group, $\mathrm{p}<0.001$. Re-resection rate due to tumour-positive margins was low in both groups; 12 patients $(4 \%)$ in the conventional resection group and 3 patients (3\%) in the oncoplastic group received a second resection. Due to study plan, patients receiving mastectomy as a second operation were excluded. In our previous study $^{18}$ of this same population including also mastectomies we have shown re-excision rate of $7.6 \%$ of which $78 \%$ were mastectomies. There was no difference in surgical complications requiring a re-operation, that is $5(2 \%)$ in the conventional group and $3(3 \%)$ in the oncoplastic group, $\mathrm{p}=0.309$. Axillary surgery, either sentinel node biopsy (SNB) or axillary lymph node dissection (ALND) was performed in 364 (96\%) patients. Axillary surgery in the oncoplastic group was more extensive than in the conventional resection group: $35(41 \%)$ received ALND in the oncoplastic group compared to 77 (26\%) in the conventional resection group, $\mathrm{p}=0.007$.

\section{Aesthetic outcome}

Aesthetic outcome according to the BCTOS questionnaire was good in $284(75 \%)$ patients. $52(61 \%)$ patients in the oncoplastic group and 230 patients $(81 \%)$ in the conventional resection group reported good aesthetic outcome, $\mathrm{p}<0.001$. The mean BCTOS aesthetic score was worse after oncoplastic resection than conventional resection (mean 1.84 vs $1.62 ; \mathrm{p}=0.002$ ). Oncoplastic resection appears to be worse in almost every BCTOS aesthetic category (Table 2).

Table 1

Patient and tumour characteristics.

\begin{tabular}{|c|c|c|c|c|}
\hline & & Conventional resection $\mathrm{N}=293$ & Oncoplastic resection $\mathrm{N}=86$ & $\mathrm{p}$-Value \\
\hline Age (median, range) & & $62(36-92)$ & $63(42-83)$ & 0.299 \\
\hline Tumour size (median, mm) & & $12.0(0.8-35)$ & $16.0(1.0-40.0)$ & $<0.001$ \\
\hline Specimen weight (g) - median (range) & & $61(11-601)$ & $97(22-1882)$ & $<0.001$ \\
\hline \multirow[t]{3}{*}{ Nodal stage } & N.A. & $18(6 \%)$ & $1(1 \%)$ & 0.029 \\
\hline & No & $210(72 \%)$ & $60(70 \%)$ & \\
\hline & N1 & $65(22 \%)$ & $26(30 \%)$ & \\
\hline \multirow[t]{4}{*}{ Histological $\mathrm{T}$ stage } & Tis \& Tmi & $19(7 \%)$ & $4(4 \%)$ & 0.016 \\
\hline & $\mathrm{T} 1$ & $240(82 \%)$ & $62(72 \%)$ & \\
\hline & $\mathrm{T} 2$ & $31(11 \%)$ & $20(23 \%)$ & \\
\hline & N.A. & $3(1 \%)$ & $0(0 \%)$ & \\
\hline \multirow[t]{4}{*}{ Histological type } & DCIS & $20(6 \%)$ & $4(4 \%)$ & 0.255 \\
\hline & Ductal & $227(74 \%)$ & $71(75 \%)$ & \\
\hline & Lobular & $30(10 \%)$ & $9(9 \%)$ & \\
\hline & Other invasive & $31(10 \%)$ & $11(11 \%)$ & \\
\hline \multirow[t]{3}{*}{ Histological grade } & 1 & $97(32 \%)$ & $24(25 \%)$ & 0.091 \\
\hline & 2 & $134(44 \%)$ & $37(39 \%)$ & \\
\hline & 3 & $75(25 \%)$ & $34(36 \%)$ & \\
\hline Multifocal & & $16(5 \%)$ & $11(12 \%)$ & 0.032 \\
\hline \multirow[t]{5}{*}{ Tumour localisation } & Central & $22(8 \%)$ & $2(2 \%)$ & 0.007 \\
\hline & Upper inner quadrant & $62(21 \%)$ & $13(15 \%)$ & \\
\hline & Lower inner quadrant & $16(5 \%)$ & $13(15 \%)$ & \\
\hline & Upper outer quadrant & $153(52 \%)$ & $41(48 \%)$ & \\
\hline & Lower outer quadrant & $40(14 \%)$ & $17(20 \%)$ & \\
\hline \multirow[t]{3}{*}{ Axillary surgery } & None & $15(5 \%)$ & 0 & 0.007 \\
\hline & SNB & $200(68 \%)$ & $51(59 \%)$ & \\
\hline & ALND & $77(26 \%)$ & $35(41 \%)$ & \\
\hline Radiation therapy & & $285(97 \%)$ & $85(99 \%)$ & 0.401 \\
\hline Radiation booster & & $45(15 \%)$ & $18(21 \%)$ & 0.225 \\
\hline
\end{tabular}

N.A.: not available, SNB: sentinel node biopsy, ALND: axillary lymph node dissection. 
Table 2

BCTOS aesthetic subscale. Individual item comparison between groups.

\begin{tabular}{llll}
\hline $\begin{array}{l}\text { No difference or } \\
\text { minimal difference in: }\end{array}$ & $\begin{array}{l}\text { Conventional } \\
\mathrm{N}=279 \\
\mathrm{~N}(\%)\end{array}$ & $\begin{array}{l}\text { Oncoplastic } \\
\mathrm{N}=86\end{array}$ & p-Value \\
\hline Breast size & $245(86 \%)$ & $60(72 \%)$ & 0.003 \\
Breast texture & $256(90 \%)$ & $65(79 \%)$ & 0.011 \\
Nipple appearance & $246(88 \%)$ & $59(75 \%)$ & 0.005 \\
Breast shape & $251(88 \%)$ & $63(76 \%)$ & 0.006 \\
Breast position & $260(92 \%)$ & $64(77 \%)$ & $<0.001$ \\
Breast Scar & $237(83 \%)$ & $59(72 \%)$ & 0.024 \\
Fit of clothing & $266(94 \%)$ & $78(93 \%)$ & 0.706 \\
\hline
\end{tabular}

According to the author-created questionnaire $217 \mathrm{pa}$ tients (75\%) in the conventional resection group and 61 patients $(72 \%)$ in the oncoplastic resection group reported good or excellent aesthetic outcome, $p=0.441$. However, the nipple position and appearance were significantly better in the conventional resection group; 112 (39\%) patients in the conventional resection group reported excellent outcome compared to 24 (29\%) in the oncoplastic group, $p=0.019$. The breast scar was also evaluated significantly better in the conventional resection group; excellent result in $115(40 \%)$ patients compared to $23(27 \%)$ patients in the oncoplastic group, $\mathrm{p}=0.040$. In breast size, position and shape there were no significant differences between groups.

\section{Factors influencing the aesthetic outcome}

Patient and tumour characteristics were analysed more closely to examine reasons for poor aesthetic outcome (Table 3). Larger tumour diameter $(\mathrm{p}=0.033)$, tumour multifocality $(\mathrm{p}=0.022)$, the weight of resection specimen $(<0.001)$ and oncoplastic surgery $(\mathrm{p}<0.001)$ were predicting poor aesthetic outcome in BCTOS aesthetic subscale, when all patients were included in the analyses. Interestingly, aesthetic outcome was not affected by tumour localization $(\mathrm{p}=0.829)$. Radiation therapy $(\mathrm{p}=0.261)$ and tumour bed booster $(p=0.231)$ had no effect on the BCTOS aesthetic outcome.

BCTOS aesthetic outcome results were analysed separately in conventional (Table 4) and oncoplastic resection (Table 5) groups. Tumour multifocality $(\mathrm{p}=0.013)$ remained predictor of poor aesthetic outcome in conventional resection group. Interestingly, it was not predictive in oncoplastic resection group: p-values 0.836 for multifocality. Tumour localization had no statistically significant effect on aesthetic outcome. In oncoplastic resection group there was a significant difference in aesthetic outcome between techniques $(\mathrm{p}=0.025)$ : extensive dual plane undermining provided more often poor aesthetic outcome than others. Reduction mammoplasty and round block techniques provided most often good aesthetic outcome.

Re-resection rate, surgical complication rate and contralateral breast symmetry procedure had no effect on BCTOS aesthetic outcome.

\section{Functional status}

Breast resection technique did not significantly affect functional status three years after surgery in the BCTOS questionnaire, BCTOS mean 1.345 in the conventional and 1.357 in the oncoplastic resection group, $\mathrm{p}=0.866$. Results were also analysed according to axillary procedure. As expected, the functional status was worse after ALND, when compared with SNB only, BCTOS mean 1.255 after SNB and 1.547 after ALND, $p<0.001$. In further analyses we did not find other factors affecting functional status, except the nodal stage, which determined the extent of axillary surgery.

\section{Discussion}

Our study results indicate that conventional resection provides good aesthetic outcome in appropriately selected patients. Patient-evaluated aesthetic outcome was good or excellent in the majority of patients.

Interestingly, on the BCTOS aesthetic subscale nearly all individual items were worse after oncoplastic resection. However, this finding is most likely due to selection bias. Oncoplastic resection allows the excision of larger or multifocal tumours to obtain best possible aesthetic outcome. Accordingly, oncoplastic resection group patients had more often larger or multifocal tumours and accordingly they received more extensive axillary surgery with earlier studies reporting similar finding. ${ }^{3}$ Larger tumour diameter and tumour multifocality had negative effect on aesthetic outcome as expected and previously reported, ${ }^{6}$ although statistically significant difference was not found when analysing oncoplastic resection group alone. Oncoplastic resection was performed more often in lower pole tumours. On contrary to earlier studies ${ }^{5-7}$ tumour location did not have an impact on aesthetic outcome.

Our results indicate that re-excision, complication surgery or radiation boost have no effect on aesthetic outcome differing from previous studies. ${ }^{6-8}$ Nevertheless, number of patients receiving complication surgery and re-excisions was low in this population, thus we consider these results unreliable and incomparable to earlier studies. ${ }^{8-10}$ Currently, radiation therapy is routinely combined with $\mathrm{BCS}$, leading to rather low number of patients not receiving radiation therapy; comparison between patients receiving radiation therapy and those few receiving BCS only is not reliable.

\section{Predictors of poor aesthetic outcome}

Contrary to previous studies, ${ }^{3,4,19}$ the tumour location did not affect the aesthetic outcome even in the conventional resection group. Successful patient selection may partly explain our better results as well as the surgical technique used; our conventional resection includes adequate mobilization and closure of breast tissue and represents 
Table 3

BCTOS aesthetic outcome. All patients.

\begin{tabular}{|c|c|c|c|c|c|}
\hline & & Good N = 284 & Intermediate $\mathrm{N}=72$ & Poor $\mathrm{N}=16$ & $\mathrm{p}$-Value \\
\hline Age & Median (range) & $62(36-83)$ & $62(36-92)$ & $63(51-70)$ & 0.783 \\
\hline \multirow[t]{7}{*}{ Breast surgery } & Conventional resection & $232(82 \%)$ & $44(61 \%)$ & $11(69 \%)$ & $<0.001$ \\
\hline & Racket & $8(3 \%)$ & $10(14 \%)$ & 0 & \\
\hline & Round block & $12(4 \%)$ & $3(4 \%)$ & $1(6 \%)$ & \\
\hline & Reduction mammoplasty & $12(4 \%)$ & $7(10 \%)$ & 0 & \\
\hline & Extensive dual plane undermining & $8(3 \%)$ & $1(1 \%)$ & $3(19 \%)$ & \\
\hline & Other & $4(1 \%)$ & 0 & 0 & \\
\hline & Rotationplasty & $8(3 \%)$ & $7(10 \%)$ & $1(6 \%)$ & \\
\hline \multirow{5}{*}{ Tumour localisation } & Central & $16(6 \%)$ & $6(8 \%)$ & $1(6 \%)$ & 0.829 \\
\hline & Upper inner & $58(20 \%)$ & $12(17 \%)$ & $5(31 \%)$ & \\
\hline & Lower inner & $23(8 \%)$ & $6(8 \%)$ & 0 & \\
\hline & Upper outer & $142(50 \%)$ & $39(54 \%)$ & $7(44 \%)$ & \\
\hline & Lower outer & $45(16 \%)$ & $9(13 \%)$ & $3(19 \%)$ & \\
\hline Tumour diameter & Median (range), $\mathrm{mm}$ & $12.0(0.8-40.0)$ & $15.0(3.0-40.0)$ & $14.0(7.0-26.0)$ & 0.033 \\
\hline \multirow[t]{2}{*}{ Multifocality } & No & $269(95 \%)$ & $65(90 \%)$ & $12(80 \%)$ & 0.022 \\
\hline & Yes & $13(5 \%)$ & $7(10 \%)$ & $3(20 \%)$ & \\
\hline \multirow[t]{4}{*}{ Histological $\mathrm{T}$ stage } & N.A. & $2(1 \%)$ & 0 & $1(8 \%)$ & 0.207 \\
\hline & $\mathrm{T} 1$ & $232(82 \%)$ & $52(72 \%)$ & $9(75 \%)$ & \\
\hline & $\mathrm{T} 2$ & $35(12 \%)$ & $14(19 \%)$ & $2(17 \%)$ & \\
\hline & Tis + T1mi & $15(5 \%)$ & $6(8 \%)$ & 0 & \\
\hline Resection specimen weight & Median, grams & 54 & 85 & 63 & $<0.001$ \\
\hline \multirow[t]{3}{*}{ Axillary surgery } & None & $9(3 \%)$ & $4(5 \%)$ & 0 & 0.271 \\
\hline & SNB & $196(69 \%)$ & $45(63 \%)$ & $8(53 \%)$ & \\
\hline & ALND & $79(28 \%)$ & $23(32 \%)$ & $7(417 \%)$ & \\
\hline Contralateral symmetry procedure & Yes & $14(5 \%)$ & $8(11 \%)$ & $1(6 \%)$ & 0.151 \\
\hline Complication surgery & Yes & $6(2 \%)$ & $2(3 \%)$ & 0 & 0.780 \\
\hline Re-resection & Yes & $9(3 \%)$ & $5(7 \%)$ & $1(6 \%)$ & 0.312 \\
\hline Radiation therapy & Yes & $280(99 \%)$ & $69(96 \%)$ & $16(100 \%)$ & 0.261 \\
\hline \multirow{3}{*}{ Tumor bed booster } & N.A. & $32(11 \%)$ & $14(19 \%)$ & $4(25 \%)$ & 0.231 \\
\hline & No & $204(72 \%)$ & $45(63 \%)$ & $10(63 \%)$ & \\
\hline & Yes & $48(17 \%)$ & $13(18 \%)$ & $2(12 \%)$ & \\
\hline
\end{tabular}

N.A.: not available, SNB: sentinel node biopsy, ALND: axillary lymph node dissection.

Table 4

Aesthetic results in conventional resection group.

\begin{tabular}{|c|c|c|c|c|c|}
\hline & & Good $N=232$ & Intermediate $\mathrm{N}=44$ & Poor $N=11$ & $\overline{p-V a l u e}$ \\
\hline Age & Median (range) & $63(36-82)$ & $61(36-92)$ & $62(52-70)$ & 0.437 \\
\hline \multirow[t]{5}{*}{ Tumour localisation } & Central & $14(6 \%)$ & $6(14 \%)$ & $1(9 \%)$ & \multirow[t]{5}{*}{0.745} \\
\hline & Upper inner & $50(22 \%)$ & $9(20 \%)$ & $3(27 \%)$ & \\
\hline & Lower inner & $14(6 \%)$ & $2(5 \%)$ & 0 & \\
\hline & Upper outer & $119(51 \%)$ & $23(52 \%)$ & $6(55 \%)$ & \\
\hline & Lower outer & $35(15 \%)$ & $4(9 \%)$ & $1(9 \%)$ & \\
\hline Tumour diameter & Median (range), $\mathrm{mm}$ & $12.0(0.8-35.0)$ & $12.5(3.0-26.0)$ & $11.0(7.0-23.0)$ & $<0.001$ \\
\hline Multifocal & Yes & $7(3 \%)$ & $4(9 \%)$ & $2(18 \%)$ & 0.013 \\
\hline \multirow[t]{4}{*}{ Histological $\mathrm{T}$ stage } & N.A. & $2(1 \%)$ & 0 & $1(9 \%)$ & \multirow[t]{4}{*}{0.489} \\
\hline & $\mathrm{T} 1$ & $192(83 \%)$ & $35(80 \%)$ & $9(82 \%)$ & \\
\hline & $\mathrm{T} 2$ & $24(10 \%)$ & $6(14 \%)$ & $1(9 \%)$ & \\
\hline & Tis + T1mi & $14(6 \%)$ & $3(7 \%)$ & 0 & \\
\hline Resection specimen weight & Median, grams & 50 & 78 & 64 & 0.586 \\
\hline \multirow[t]{3}{*}{ Axillary surgery } & None & $9(4 \%)$ & $4(9 \%)$ & 0 & \multirow[t]{3}{*}{0.090} \\
\hline & SNB & $166(71 \%)$ & $28(64 \%)$ & $4(40 \%)$ & \\
\hline & ALND & $57(25 \%)$ & $12(27 \%)$ & $6(60 \%)$ & \\
\hline Contralateral symmetry procedure & Yes & 0 & $2(5 \%)$ & $1(9 \%)$ & 0.001 \\
\hline Complication surgery & Yes & $3(1 \%)$ & $2(5 \%)$ & 0 & 0.293 \\
\hline Re-resection & Yes & $8(3 \%)$ & $3(7 \%)$ & $1(9 \%)$ & 0.420 \\
\hline Radiation therapy & Yes & $229(99 \%)$ & $41(93 \%)$ & $11(100 \%)$ & 0.056 \\
\hline \multirow[t]{3}{*}{ Tumour bed booster } & N.A. & $29(13 \%)$ & $10(23 \%)$ & $3(27 \%)$ & \multirow[t]{3}{*}{0.296} \\
\hline & No & $167(72 \%)$ & $27(61 \%)$ & $6(55 \%)$ & \\
\hline & Yes & $36(15 \%)$ & $7(16 \%)$ & $2(18 \%)$ & \\
\hline
\end{tabular}

N.A.: not available, SNB: sentinel node biopsy, ALND: axillary lymph node dissection. 
Table 5

Aesthetic results in oncoplastic resection group.

\begin{tabular}{|c|c|c|c|c|c|}
\hline & & Good $\mathrm{N}=52$ & Intermediate $\mathrm{N}=28$ & Poor $\mathrm{N}=5$ & $\mathrm{p}$-Value \\
\hline Age & median (range) & $62(42-83)$ & $64(46-76)$ & $63(51-68)$ & 0.642 \\
\hline \multirow[t]{6}{*}{ Breast surgery } & Racket & $8(15 \%)$ & $10(36 \%)$ & 0 & 0.025 \\
\hline & Round block & $12(23 \%)$ & $3(11 \%)$ & $1(20 \%)$ & \\
\hline & Reduction mammoplasty & $12(23 \%)$ & $7(25 \%)$ & 0 & \\
\hline & Extensive dual plane undermining & $8(15 \%)$ & $1(4 \%)$ & $3(60 \%)$ & \\
\hline & Other & $4(8 \%)$ & 0 & 0 & \\
\hline & Rotationplasty & $8(15 \%)$ & $7(25 \%)$ & $1(20 \%)$ & \\
\hline \multirow[t]{5}{*}{ Tumour localisation } & Central & $2(14 \%)$ & 0 & 0 & 0.524 \\
\hline & Upper inner & $8(15 \%)$ & $3(11 \%)$ & $2(40 \%)$ & \\
\hline & Lower inner & $9(17 \%)$ & $4(14 \%)$ & 0 & \\
\hline & Upper outer & $23(44 \%)$ & $16(57 \%)$ & $1(20 \%)$ & \\
\hline & Lower outer & $10(19 \%)$ & $5(18 \%)$ & $2(40 \%)$ & \\
\hline Tumour diameter & Median (range), $\mathrm{mm}$ & $16.0(11.0-40.0)$ & $17.5(5.0-40.0)$ & $15.0(9.0-26.0)$ & 0.255 \\
\hline Multifocality & Yes & $6(12 \%)$ & $3(11 \%)$ & $1(20 \%)$ & 0.836 \\
\hline \multirow[t]{3}{*}{ Histological $\mathrm{T}$ stage } & $\mathrm{T} 1$ & $40(77 \%)$ & $17(61 \%)$ & $4(80 \%)$ & 0.512 \\
\hline & $\mathrm{T} 2$ & $11(21 \%)$ & $8(28 \%)$ & $1(20 \%)$ & \\
\hline & Tis + T1mi & $1(2 \%)$ & $3(11 \%)$ & 0 & \\
\hline Resection specimen weight & Median, grams & 91 & 95 & 62 & 0.674 \\
\hline \multirow[t]{2}{*}{ Axillary surgery } & SNB & $30(58 \%)$ & $17(61 \%)$ & $4(80 \%)$ & 0.949 \\
\hline & ALND & $22(42 \%)$ & $11(39 \%)$ & $1(20 \%)$ & \\
\hline Contralateral symmetry procedure & Yes & $14(27 \%)$ & $6(12 \%)$ & 0 & 0.379 \\
\hline Complication surgery & Yes & $3(6 \%)$ & 0 & 0 & 0.384 \\
\hline \multirow[t]{2}{*}{ Re-resection } & No & $51(98 \%)$ & $26(93 \%)$ & $5(100 \%)$ & 0.438 \\
\hline & Yes & $1(2 \%)$ & $2(7 \%)$ & 0 & \\
\hline Radiation therapy & Yes & $51(98 \%)$ & $28(100 \%)$ & $5(100 \%)$ & 0.725 \\
\hline \multirow{3}{*}{ Tumour bed booster } & N.A. & $3(6 \%)$ & $4(14 \%)$ & $1(20 \%)$ & 0.494 \\
\hline & No & $37(71 \%)$ & $18(64 \%)$ & $4(80 \%)$ & \\
\hline & Yes & $12(23 \%)$ & $6(21 \%)$ & 0 & \\
\hline
\end{tabular}

N.A.: not available, SNB: sentinel node biopsy, ALND: axillary lymph node dissection.

actually level 1 oncoplastic surgery. Larger resection specimen weight appears to be a predictor of poor aesthetic outcome in this study as well as the in previous reports, ${ }^{5}$ although Rezai et al. reported opposite results. ${ }^{19}$ However, we have no data on the tumour/breast volume ratio, which might better represent the extent of resection than resection specimen weight alone. More extensive remodelling of breast tissue, performed in oncoplastic surgery, is possibly leading to notable difference in breast firmness and shape. Many oncoplastic techniques cause more visible and longer scars and include centralization of the nipple areolacomplex, which may affect patient's appraisal.

Moreover, patients receiving oncoplastic resection have no experience of conventional resection, which in these larger tumours might result in breast deformity, possibly influencing the evaluation of the aesthetic outcome. Similar phenomenon can be seen in breast reconstruction after mastectomy: patient's satisfaction is lower after immediate breast reconstruction than after delayed breast reconstruction when patient has been living without a breast for a period of time. ${ }^{20}$

\section{Questionnaires, evaluation of aesthetic outcome}

Even though aesthetic outcome is an essential quality indicator and important endpoint in breast cancer surgery, treatment outcome measurement and especially surgical issues after BCT are lacking standardized evaluation methods. Wide variation exists in methodology of previous studies evaluating aesthetic outcome after BCT making comparison of results difficult. ${ }^{1,3,4}$ A systematic review by Chen et al. underlines the need for better evaluation tools. ${ }^{15}$ When evaluating treatment outcome, patient selfevaluation is a valuable method since the subjective experience is central to assessment of QoL. ${ }^{2,16}$

The most appropriate time to evaluate aesthetic outcome after BCT and irradiation therapy is at least two years postoperatively, due to long-term effects of irradiation. ${ }^{8,13}$ Fibrosis related to irradiation can increase up to three years. ${ }^{7}$ Previous studies concerning aesthetic outcome after BCT and oncoplastic surgery also differ greatly regarding the evaluation time-point of aesthetic outcome, varying from immediate post-operative time ${ }^{4,6}$ to several years after surgery. ${ }^{7}$ We evaluated aesthetic outcome three years after surgery, thus sufficient time after radiation therapy has passed to visualize effects of irradiation and booster.

\section{Limitations}

The major limitation of the study was the selection bias favouring the conventional resection group. Also the number of patients in the oncoplastic group was rather low. Several oncoplastic techniques were used and the number of patients having each technique used was small. 
Therefore some significant associations may have remained undetected.

Moreover, the study period coincided with the beginning of oncoplastic era in our unit and our breast surgeons have currently more experience in oncoplastic surgery. Accordingly, both patient selection for oncoplastic resection and selection of the oncoplastic techniques may have improved.

\section{Conclusions}

Overall patient satisfaction with aesthetic outcome after BCT is high. Conventional resection provides good aesthetic outcome in appropriately selected patients. Oncoplastic resection enables BCT in patients with larger and multifocal tumours with a favourable aesthetic outcome.

\section{Conflict of interest}

None.

\section{Acknowledgements}

KO was supported by Kurt and Doris Palander Foundation grant.

\section{References}

1. Heil J, Czink E, Golatta M, et al. Change of aesthetic and functional outcome over time and their relationship to quality of life after breast conserving therapy. Eur J Surg Oncol (EJSO) 2011;37:116-21.

2. Heil J, Holl S, Golatta M, et al. Aesthetic and functional results after breast conserving surgery as correlates of quality of life measured by a German version of the breast cancer treatment outcome scale (BCTOS). Breast 2010;19:470-4.

3. Santos G, Urban C, Edelweiss MI, et al. Long-term comparison of aesthetical outcomes after oncoplastic surgery and lumpectomy in breast cancer patients. Ann Surg Oncol 2015;22:2500-8.

4. Foersterling E, Golatta M, Hennigs A, et al. Predictors of early poor aesthetic outcome after breast-conserving surgery in patients with breast cancer: initial results of a prospective cohort study at a single institution. J Surg Oncol 2014;110:801-6.

5. Hennigs A, Hartmann B, Rauch G, et al. Long-term objective esthetic outcome after breast-conserving therapy. Breast Cancer Res Treat 2015;153:345-51.
6. Heil J, Breitkreuz K, Golatta M, et al. Do reexcisions impair aesthetic outcome in breast conservation surgery? Exploratory analysis of a prospective cohort study. Ann Surg Oncol 2012;19:541-7.

7. Immink JM, Putter H, Bartelink H, et al. Long-term cosmetic changes after breast-conserving treatment of patients with stage I-II breast cancer and included in the EORTC 'boost versus no boost' trial. Ann Oncol 2012;23:2591-8.

8. Vrieling C, Collette L, Fourquet A, et al. The influence of patient, tumor and treatment factors on the cosmetic results after breastconserving therapy in the EORTC 'boost vs. no boost' trial. Radiotherapy Oncol 2000;55:219-32.

9. Chakravorty A, Shrestha AK, Sanmugalingam N, et al. How safe is oncoplastic breast conservation?: comparative analysis with standard breast conserving surgery. Eur J Surg Oncol (EJSO) 2012;38:395-8.

10. Losken A, Dugal CS, Styblo TM, Carlson GW. A meta-analysis comparing breast conservation therapy alone to the oncoplastic technique. Ann Plast Surg 2014;72:145-9.

11. Rezai M, Kraemer S, Kimmig R, Kern P. Breast conservative surgery and local recurrence. Breast 2015;24(Suppl. 2):S100-7.

12. De Lorenzi F, Hubner G, Rotmensz N, et al. Oncological results of oncoplastic breast-conserving surgery: long term follow-up of a large series at a single institution: a matched-cohort analysis. Eur J Surg Oncol 2016 Jan;42(1):71-7.

13. Haloua MH, Krekel NM, Winters HA, et al. A systematic review of oncoplastic breast-conserving surgery: current weaknesses and future prospects. Ann Surg 2013;257:609-20.

14. Lansu JT, Essers M, Voogd AC, et al. The influence of simultaneous integrated boost, hypofractionation and oncoplastic surgery on cosmetic outcome and PROMs after breast conserving therapy. Eur J Surg Oncol 2015;41:1411-6.

15. Chen CM, Cano SJ, Klassen AF, et al. Measuring quality of life in oncologic breast surgery: a systematic review of patient-reported outcome measures. Breast J 2010;16:587-97.

16. Stanton AL, Krishnan L, Collins CA. Form or function? Part 1. subjective cosmetic and functional correlates of quality of life in women treated with breast-conserving surgical procedures and radiotherapy. Cancer 2001;91:2273-81.

17. Clough KB, Kaufman GJ, Nos C, Buccimazza I, Sarfati IM. Improving breast cancer surgery: a classification and quadrant per quadrant atlas for oncoplastic surgery. Ann Surg Oncol 2010;17: 1375-91.

18. Ojala K, Meretoja TJ, Mattson J, et al. The quality of preoperative diagnostics and surgery and their impact on delays in breast cancer treatment - a population based study. Breast 2016;26:80-6.

19. Rezai M, Knispel S, Kellersmann S, Lax H, Kimmig R, Kern P. Systematization of oncoplastic surgery: selection of surgical techniques and patient-reported outcome in a cohort of 1,035 patients. Ann Surg Oncol 2015;22:3730-7.

20. Meretoja TJ, Svarvar C, Jahkola TA. Outcome of oncoplastic breast surgery in 90 prospective patients. Am J Surg 2010;200:224-8. 